\begin{tabular}{llll}
\hline Madera y Bosques vol. 25, núm. 2, e2521773 & Verano 2019 & Artículos científicos
\end{tabular}

\title{
Respuesta climática de Abies guatemalensis Rehder en Ixtlán de Juárez, Oaxaca, México
}

\author{
Climatic response of Abies guatemalensis Rehder in Ixtlán de Juarez, \\ Oaxaca, Mexico
}

\author{
Martín Aquino-Ramírez', Alejandro Velázquez-Martínez", José Villanueva-Díaz², Haidie Lissette Hervert-Zamora³, \\ Armando Gómez-Guerrero', Valentín José Reyes-Hernández' y Gustavo Ramírez-Valverde ${ }^{4}$
}

\begin{abstract}
Colegio de Postgraduados. Postgrado en Ciencias Forestales. Montecillo, Texcoco, Estado de México.

2 Instituto Nacional de Investigaciones Forestales Agrícolas y Pecuarias. Centro Nacional de
\end{abstract}

\author{
Investigación Disciplinaria en Relación Agua, Suelo, \\ Planta, Atmósfera. Gómez Palacio, Durango, México \\ 3 Universidad Politécnica de Texcoco. San Miguel \\ Coatlinchán, Texcoco, Estado de México.
}

\author{
${ }^{4}$ Colegio de Postgraduados. Postgrado en Socio- \\ economía y Estadística. Montecillo, Texcoco, Estado \\ de México \\ *Autor de correspondencia. alejvela@colpos.mx
}

\section{RESUMEN}

En México existen especies arbóreas, específicamente, el grupo de las coníferas, las cuales permiten analizar y reconstruir las variaciones climáticas del pasado de una o varias regiones geográficas. El objetivo de este estudio fue determinar el potencial de respuesta dendrocronológica de Abies guatemalensis Rehder a la variabilidad climática en Ixtlán de Juárez, Oaxaca. Se desarrollaron cronologías de madera temprana, de madera tardía y de anillo total de 206 años (1810-2015). El análisis de correlación estacional señaló una relación directa entre la cronología RWI residual con la precipitación de invierno-primavera $(\mathrm{r}=0.67, \mathrm{p}<0.01)$ y una relación indirecta con la temperatura máxima de primavera $(\mathrm{r}=-0.42, \mathrm{p}<0.01)$ del año actual de crecimiento. Mediante un modelo de regresión lineal se reconstruyó la precipitación acumulada de febrero a abril, de los últimos 136 años (1880-2015), el modelo explicó 44\% de la variabilidad de los registros meteorológicos locales del período de calibración (1952-2010). Los períodos más secos ocurrieron en las décadas 1880, 1900, 1940, 1970, 1980, 1990 y 2000. El análisis espectral de la reconstrucción mostró una periodicidad cuasi-cíclica de 2.2 años y 3.8 años, indicando una asociación con el fenómeno ENSO. La sensibilidad de la especie a variaciones climáticas reflejadas en el grosor de los anillos de crecimiento sugiere que $A$. guatemalensis tiene potencial para reconstrucciones hidroclimáticas, lo que permitirá extender en el tiempo los registros instrumentales para análisis más amplios de fluctuaciones en el clima y sus tendencias en esta región del país.

PALABRAS CLAVE: dendrocronología, ENSO, oyamel, precipitación, variabilidad climática.

\section{ABSTRACT}

In Mexico there are tree species, specifically, the group of conifers, which allow to analyze and reconstruct the climatic variations of the past of one or more geographical regions. The objective of this study was to determine the dendrochronological response potential of Abies guatemalensis Rehder. to climatic variability in Ixtlán de Juárez, Oaxaca. Earlywood, latewood and total ring chronologies of 206 years (1810-2015) were developed. The seasonal correlation analysis revealed a direct relationship between the residual RWI chronology with the winter-spring precipitation $(\mathrm{r}=0.67, \mathrm{p}<0.01)$ and an indirect relationship with the maximum spring temperature $(\mathrm{r}=-0.42, \mathrm{p}$ $<0.01$ ) of the current year of growth. Using a linear regression model, the accumulated precipitation from February to April was reconstructed over the last 136 years (1880-2015), which explained $44 \%$ of the variability of the local meteorological records of the calibration period (1952-2010). The driest periods occurred in the 1880's, 1900's, 1940's, 1970's, 1980's, 1990's and 2000's. The spectral analysis of the reconstruction showed quasi-cyclic periodicity of 2.2 years and 3.8 years, indicating an association with El Niño-Southern Oscillation. The sensitivity of the species to climatic variations reflected in the thickness of its growth rings, suggests that $A$. guatemalensis has potential for hydroclimatic reconstructions, which will allow to extend in time the instrumental records for broader analyses of fluctuations in the climate and its trends in this region of the country.

KEYWORDS: dendrochronology, ENSO, fir, precipitation, climatic variability. 


\section{INTRODUCCIÓN}

Los anillos de crecimiento de los árboles son equivalentes a archivos climáticos naturales con información sobre el clima y el ambiente donde se desarrollan, pudiendo abarcar siglos o incluso milenios (Zhang, 2015). Las cronologías generadas con los anillos de crecimiento de los árboles han permitido ampliar, mejorar o sustituir los registros meteorológicos respecto a los registros instrumentales. Las series dendrocronológicas también permiten analizar las variaciones climáticas interanuales o multianuales, definir tendencias y modelar el impacto del cambio climático en el crecimiento arbóreo. Las principales ventajas del uso de los anillos de crecimiento de los árboles en las reconstrucciones climáticas comparado con otras fuentes indirectas de información del clima como capas de hielo, sedimentos marinos, polen, arrecifes coralinos, entre otros, es que estos presentan una amplia distribución espacial, de resolución anual y son sensibles a la variación estacional, anual o multianual del clima (Frank, Esper, Zorita y Wilson, 2010).

Los estudios dendroclimáticos en México se encargan de reconstruir el clima local y regional del pasado (Villanueva et al., 2015) y su conexión con patrones climáticos de escala global como El Niño Oscilación del Sur (ENSO, por sus siglas en inglés), la Oscilación Decadal del Pacífico (PDO, por sus siglas en inglés) y la Oscilación Multidecadal del Pacífico (PDO, por sus siglas en inglés); asimismo, identifican y analizan eventos hidroclimáticos históricos (Stahle et al., 2016).

Las especies frecuentemente utilizadas para las reconstrucciones del clima en México han sido Pseudotsuga meñiesii (Mirb.) Franco, Taxodium mucronatum Ten. y Pinus hartwegii Lindl.; en las regiones del norte-centro y, en un menor grado, en la región de sur del país (AcostaHernández, Pompa-García y Camarero, 2017). Exhibiendo así una necesidad de incorporar nuevas especies a los estudios climáticos y desarrollar una red dendrocronológica en el sur de México, donde los estudios dendrocronológicos son escasos, particularmente para el género Abies (BernalSalazar, Terrazas y Alvarado, 2004).
En los bosques de coníferas a lo largo del occidente y sur de México, se encuentran pequeñas poblaciones aisladas y restringidas de Abies guatemalensis Rehder, localizadas en laderas y cañadas. Esta especie se desarrolla bajo condiciones de alta humedad y con temperaturas anuales medias $>11^{\circ} \mathrm{C}$ (Islebe, Velázquez y Cleef, 1995; Ramírez y González, 2015), de manera tal, que su crecimiento está sujeto a condiciones muy particulares, que lo hacen sensible a variaciones climáticas. Por ejemplo, Anchukaitis et al. (2013) mencionan que el crecimiento de $A$. guatemalensis en la sierra de los Cuchumatanes, Guatemala es afectado por las condiciones de humedad al inicio de la estación de crecimiento, lo que es controlado por la precipitación al final de la estación seca, pero su crecimiento se ve negativamente influenciado por la temperatura de la estación de crecimiento, demostrando así, el potencial dendroclimático de la especie.

Por lo anterior, es necesario desarrollar estudios dendroclimáticos para analizar la influencia de las condiciones específicas del clima en el crecimiento de $A$. guatemalensis y generar series dendrocronológicas, que permitan reconstruir la variabilidad climática del pasado, más allá de la disponibilidad de datos climáticos instrumentales, específicamente para el estado de Oaxaca, en donde estos registros son de corta extensión ( $<60$ años) (Pérez-Morga et al., 2008). El entendimiento de las variaciones climáticas a diferentes escalas espaciotemporales y sus efectos en las limitaciones ambientales de los ecosistemas forestales, permitirá mejorar la predicción de respuestas a los cambios futuros.

\section{OBjetIVOS}

Los objetivos planteados en este trabajo fueron: a) evaluar el potencial dendrocronológico de $A$. guatemalensis, b) analizar la influencia del clima local en el crecimiento radial, y c) reconstruir la precipitación histórica para analizar su variabilidad y determinar la influencia en sus comportamiento de fenómenos circulatorios atmosféricos a gran escala (ENSO, PDO y AMO) para la zona de Ixtlán 
de Juárez, Oaxaca, bajo las siguientes hipótesis: 1) el principal factor limitante en el crecimiento radial es la precipitación, factor que permitirá reconstruir y caracterizar la variabilidad climática histórica del área de estudio, y 2) la variabilidad de la precipitación es influenciada por los eventos intensos de ENSO.

\section{MATERIALES Y MÉTODOS}

\section{Área de estudio}

El estudio se realizó en la región de la Sierra Norte de Oaxaca, en el municipio de Ixtlán de Juárez. Se seleccionaron dos sitios donde $A$. guatemalensis se distribuye de manera natural localizados en las coordenadas geográficas $17^{\circ} 28^{\prime} 28.12^{\prime \prime}$ y $17^{\circ} 28^{\prime} 18.96$ ” latitud norte y los $96^{\circ} 24^{\prime} 54.43^{\prime \prime}$ O y $96^{\circ} 24^{\prime} 47.78^{\prime \prime}$ longitud oeste, a una altitud de $2200 \mathrm{~m}$ a $2400 \mathrm{~m}$ snm (Fig. 1). El tipo de clima de los sitios de estudio corresponde a templado húmedo con lluvias de verano C (m) (García, 2004). La geomorfología de la zona es de serranía con tipo de suelo Acrisol (Instituto Nacional de Estadística y Geografía [Inegi], 2010). La población de A. guatemalensis se encontró en el bosque de pino-encino, asociado con Pinus patula Schltdl. \& Cham., Pinus ayacabuite Ehrenb. ex Schltdl., Quercus candicans Née, Litsea neesiana (S. Schauer) Hemsl., Litsea glaucescens Kunth y Phoebe helicterifolia (Meisn.) Mez.

\section{Muestreo}

Los árboles de A. guatemalensis seleccionados fueron aquellos que presentaron un disturbio menor y se apreciaron libres de daños físicos y/o enfermedades. Con ayuda de un taladro de Pressler marca Haglöf de $12 \mathrm{~mm}$ de diámetro interno y $53.34 \mathrm{~cm}$ de longitud, se obtuvieron de 2 a 3 núcleos de crecimiento por individuo, a una altura de $1.30 \mathrm{~m}$ con respecto al suelo y en dirección perpendicular a la pendiente, con el fin de evitar anomalías en el crecimiento (problemas de tensión y compresión). Se colectó un total de 74 muestras correspondientes a 35 árboles.

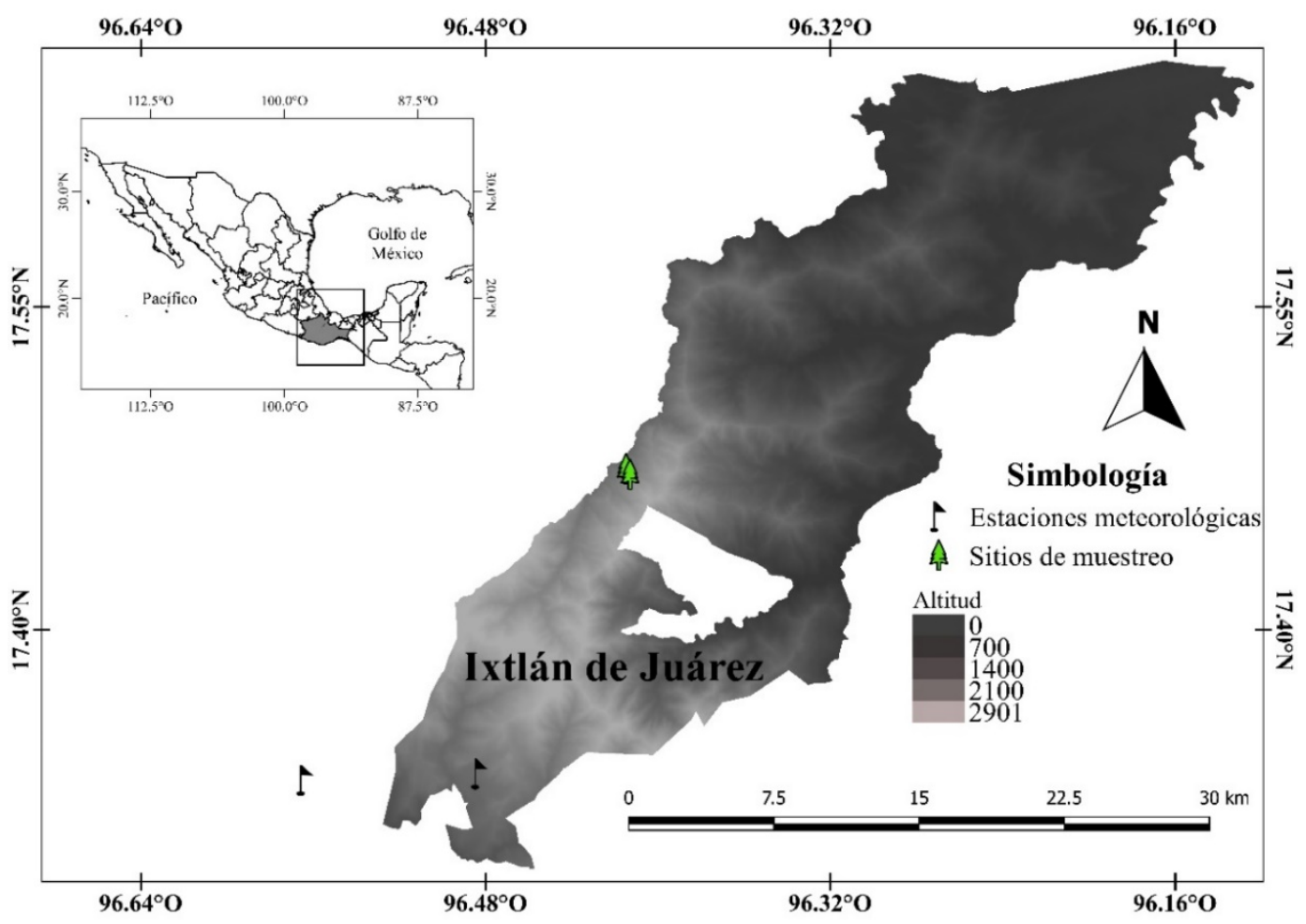

FIGURA 1. Localización geográfica del área de estudio y las estaciones meteorológicas más cercanas. 
Las muestras se secaron a temperatura ambiente y se colocaron en moldes de madera donde fueron lijadas y pulidas utilizando papel lija (grano grueso 200 a fino 1000) para mejorar la visibilidad entre límites de los anillos de crecimiento. El prefechado de las muestras se realizó de acuerdo con procedimientos dendrocronológicos tradicionales (Speer, 2010). El anillo anual, la madera temprana y la madera tardía, se midieron con un sistema de medición Velmex, con una precisión $0.001 \mathrm{~mm}$. La calidad del fechado se realizó en el programa COFECHA (Holmes, 1983).

\section{Estandarización y desarrollo de la cronología}

Las series fueron estandarizadas empleando el paquete dplR (Bunn, 2010) de los programas dendrocronológicos de la Universidad de Arizona en el software R (R Development Core Team, 2017). Se ajustó un spline cúbico suavizado con $50 \%$ de frecuencia de respuesta y $67 \%$ de la longitud de la serie para remover las tendencias biológicas y geométricas de cada serie y resaltar la señal climática (Cook, 1987). La estandarización de las series individuales con media igual a 1.0 y varianza homogénea se obtuvo dividiendo la medición de ancho de anillo de cada año entre el valor de la curva de ajuste (Cook, 1987). Siguiendo este procedimiento, se generó una cronología promedio estandarizada para la madera temprana (EWI), la madera tardía (LWI) y el anillo total (RWI). Las tres cronologías incluyeron las versiones estándar (STD) y residual (RES). Para determinar la calidad y confiabilidad de las cronologías, se calculó la señal expresada de la población (EPS) (Wigley, Briffa y Jones, 1984). El EPS indica el grado en que el tamaño de la muestra con que se generó la cronología es representativo de una población teórica, con un número infinito de individuos. Una cronología con un valor EPS $>0.85$ es considerado confiable para reconstrucciones climáticas (Wigley et al., 1984).

\section{Datos climáticos}

Los registros de precipitación promedio (Prec), temperatura máxima (Tmáx), temperatura promedio
(Tprom) y temperatura mínima (Tmín) fueron obtenidos del programa Extractor Rápido de Información Climatológica III (ERIC III) (Instituto Mexicano de Tecnología del Agua [IMTA], 2013). Las estaciones meteorológicas más cercanas a los sitios de muestreo fueron: Ixtlán de Juárez $\left(96.48^{\circ}\right.$ latitud norte y $17.33^{\circ}$ longitud oeste a altitud de $2312 \mathrm{~m} \mathrm{snm}$ ) y Guelatao $\left(96.564^{\circ}\right.$ latitud norte y $17.330^{\circ}$ longitud oeste a altitud de $1496 \mathrm{~m}$ $\mathrm{snm})$ con una distancia de $17.5 \mathrm{~km}$ y $23 \mathrm{~km}$, respectivamente (Fig. 1). Los registros meteorológicos de las dos estaciones fueron promediados para construir una base local abarcando un período de 1952-2010, que se utilizó para el análisis de correlación con el crecimiento radial del árbol. La precipitación total anual es de $989 \mathrm{~mm}$, registrándose la mayor cantidad en el mes de septiembre. La temperatura promedio anual es de $13.5^{\circ} \mathrm{C}$, registrando el valor más bajo en los meses de diciembre-febrero y los valores más altos en el mes de abril (Fig. 2).

\section{Análisis estadístico}

La respuesta climática de $A$. guatemalensis fue analizada mediante una correlación lineal de Pearson's entre los índices de ancho de anillo de EWI, LWI y RWI con las variables Prec, Tmáx, Tprom y Tmín, para determinar qué variables climáticas estaban más relacionadas con el crecimiento radial en el sitio de estudio. El período de

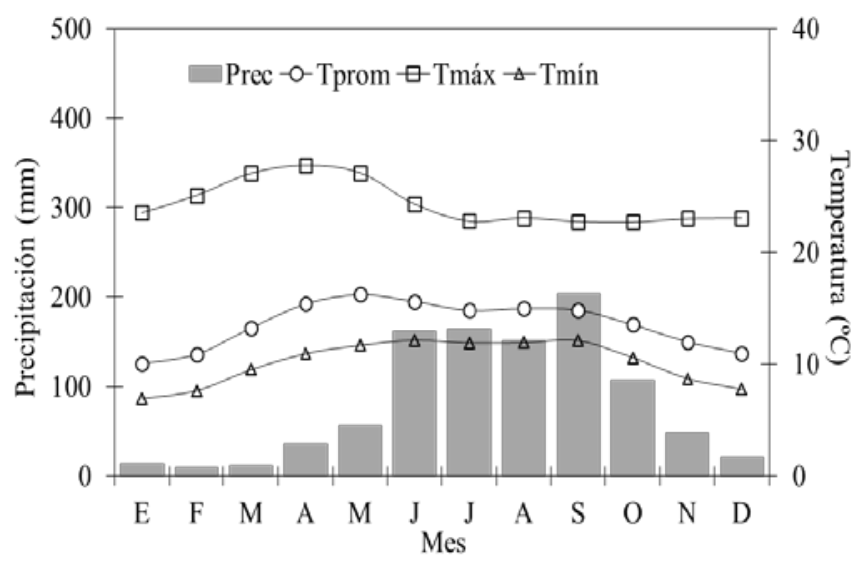

FIGURA 2. Registros promedios mensuales de precipitación (Prec), temperatura máxima (Tmáx), temperatura promedio (Tprom) y temperatura mínima (Tmín) del período 1952-2010, para las estaciones meteorológicas Ixtlán de Juárez y Guelatao, Oaxaca. 
análisis se realizó adoptando el año biológico clásico, es decir, bajo el supuesto que el crecimiento de los árboles puede ser afectado por las condiciones climáticas prevalecientes de octubre del año previo a septiembre del año actual de crecimiento (Gaire et al., 2017). Las correlaciones mensuales y estacionales de los datos climáticos se realizaron con la función seascorr en el paquete treeclim (Zang y Biondi, 2015) del software R. Este análisis permitió calcular intervalos de confianza para las correlaciones a través de un remuestreo bootstrap estacionario (1000 repeticiones) con un nivel de confiabilidad de $95 \%$ y $99 \%$.

Posteriormente, se ajustó un modelo de regresión lineal con la función skills del paquete treeclim para reconstruir la variable climática que mostró mayor influencia en la variabilidad del crecimiento radial de $A$. guatemalensis. Para ello, los registros meteorológicos instrumentales fueron divididos en dos subperíodos de la misma longitud; uno para la calibración y otro para la verificación. Si los dos subperíodos fueran significativos, entonces se utilizará el período total de los registros instrumentales para ajustar el modelo de reconstrucción final. La confiabilidad del modelo fue evaluado con el coeficiente de regresión $\left(R^{2}\right)$, el coeficiente de regresión ajustado $\left(R_{a d j}\right)$, el coeficiente de correlación ( $r$ ) y la significancia del modelo (F); y la capacidad de predicción del modelo con la reducción del error (RE), el coeficiente de eficiencia (CE), la raíz de los cuadrados medios del error (RMSE) y el estadístico Durbin Watson (DW). Los valores positivos de RE y CE indican que el modelo de regresión tiene potencial para reconstrucciones climáticas (Cook, Meko, Stahle y Cleaveland, 1999).

Para analizar los cambios en la reconstrucción climática, los años secos extremos se identificaron como aquellos años menores a la media -1 desviación estándar $(\sigma)$ y los años húmedos extremos mayores a la media +1 desviación estándar $(\sigma)$, en tanto que, dos o más años consecutivos abajo de la media se consideraron como sequía o húmedos aquellos por arriba de la media, de acuerdo con el método de división utilizado por Sun et al.
(2018). Así mismo, la variabilidad climática de la serie de reconstrucción en la frecuencia dominante fue examinada con un análisis espectral, para detectar la periodicidad de eventos originados por fenómenos circulatorios (Mann y Lees, 1996).

La influencia de fenómenos circulatorios atmosféricos en el crecimiento de los árboles fue evaluada con una función de correlación para contrastar las cronologías de índices de ancho de anillo de $A$. guatemalensis con los índices del El Niño-Oscilación del Sur (ENSO; Ropelewski y Halpert, 1987), Oscilación Decadal del Pacífico (PDO; Mantua et al., 1997) y Oscilación Multidecadal del Atlántico (AMO; Van Oldenborgh et al., 2009). El índice multivariado del ENSO (MEI), el índice oscilación del sur (SOI), el índice Niño 3.4 SST y el índice de lluvia tropical (TRI) fueron analizados como indicadores de la variabilidad del ENSO. El MEI es una serie de tiempo bimensual que permite caracterizar los eventos de ENSO, se fundamenta en seis variables registradas en el Pacífico tropical: presión al nivel del mar, componentes de la superficie del viento meridional y zonal, temperatura del agua y del aire y la fracción de la nubosidad total del cielo (Wolter y Timlin, 1998); el SOI es un índice estandarizado basado en las diferencias de presión observadas a nivel del mar entre Tahití y Darwin, Australia (Ropelewski y Jones, 1987); el índice Niño 3.4 SST define las anomalías promedio de la temperatura de la superficie del mar en la franja comprendida entre $5^{\circ} \mathrm{N}-5^{\circ} \mathrm{S}$ y $120^{\circ}-170^{\circ} \mathrm{W}$ (Rayner et al., 2003) y el TRI constituye una estimación de la variabilidad de ENSO, ya que está relacionado con las anomalías en la cantidad de lluvia en el Océano Pacífico tropical (Wright, 1979). Por otro lado, el índice PDO indica las variaciones mensuales de la temperatura superficial marina en el Océano Pacífico al norte de los $20^{\circ} \mathrm{N}$ que cambia a escala interdecadal en períodos aproximados de 20 años a 30 años (Hare y Mantua, 2000) y el índice AMO describe la variabilidad climática multidecadal en el Océano Atlántico norte (van Oldenborgh et al., 2009). Las series de tiempo mensuales de los índices MEI, SOI, Niño 3.4 SST, TRI, PDO y AMO fueron obtenidas de la base de datos del 
National Oceanic and Atmospheric Administration (NOAA) Earth System Research Laboratory (https://www.esrl.noaa.gov/psd/data/climateindices/list /). Todos los análisis estadísticos mencionados anteriormente se realizaron con el software R versión 3.4.2 (R Development Core Team, 2017).

\section{Resultados}

\section{Cronología}

Se fecharon correctamente $62(84 \%)$ de las muestras de $A$. guatemalensis. El ancho de anillo total promedio fue de 3.40 $\mathrm{mm} \pm 0.24 \mathrm{~mm}$. Los anillos fueron relativamente grandes en la mayoría de las muestras, lo que facilitó el fechado y la presencia de anillos falsos fue común en los últimos años. La longitud de las series individuales abarcó de 206 años a 71 años con una media de 115 años en el período de 18102015. La cronología residual de anillo total presentó una intercorrelación entre series de 0.57 ( $p<0.01$ ), valor que supera al de referencia 0.3281 ( $\mathrm{p}<0.01$ ) (Holmes, 1983); sensibilidad media de 0.31, desviación estándar de 0.19, autocorrelación de primer orden de 0.42 , relación señalruido de 13.77 y varianza en el primer "eigenvector" de $38.83 \%$. La longitud entre series individuales abarcó de 206 años a 71 años con una media de 115 años en el período de 1810-2015. La confiabilidad de la cronología se presentó a partir de 1880 cuando el valor del EPS (0.93) superó el umbral recomendado de 0.85 (Wigley et al., 1984), con 10 radios en adelante (Fig. 3),

\section{Respuesta climática}

Los resultados del análisis de correlación indicaron que la precipitación del mes de abril presentó una relación positiva con la cronología residual de anillo total $(r=0.66, p<0.01)$, madera temprana $(\mathrm{r}=0.61, \mathrm{p}<0.05)$ y tardía $(\mathrm{r}=0.41, \mathrm{p}$ $<0.05$ ) (Tabla 1). De igual manera, la precipitación estacional acumulada de marzo-abril presentó una correlación positiva con la cronología residual de madera temprana $(\mathrm{r}=0.60, \mathrm{p}<0.05)$ y con la cronología estándar de madera tardía $(r=0.60, \mathrm{p}<0.05)$. Las correlaciones negativas significativas fueron entre la cronología residual de ancho de anillo total y la temperatura promedio de marzo ( $\mathrm{r}=-0.29, \mathrm{p}<0.01)$, abril $(\mathrm{r}=-0.39, \mathrm{p}<0.01)$, mayo $(\mathrm{r}=-0.28, \mathrm{p}<0.01)$ y estacionalmente con los meses de febrero-abril $(\mathrm{r}=-0.32, \mathrm{p}<0.01)$ del año actual de crecimiento (Fig. 4). La correlación entre la cronología residual de madera temprana y la temperatura promedio solo fue negativa con el mes de noviembre previo a la estación de crecimiento $(\mathrm{r}=-0.28, \mathrm{p}<0.05)$; mientras que para la cronología estándar de madera tardía, la correlación fue positiva con la temperatura máxima del mes de agosto

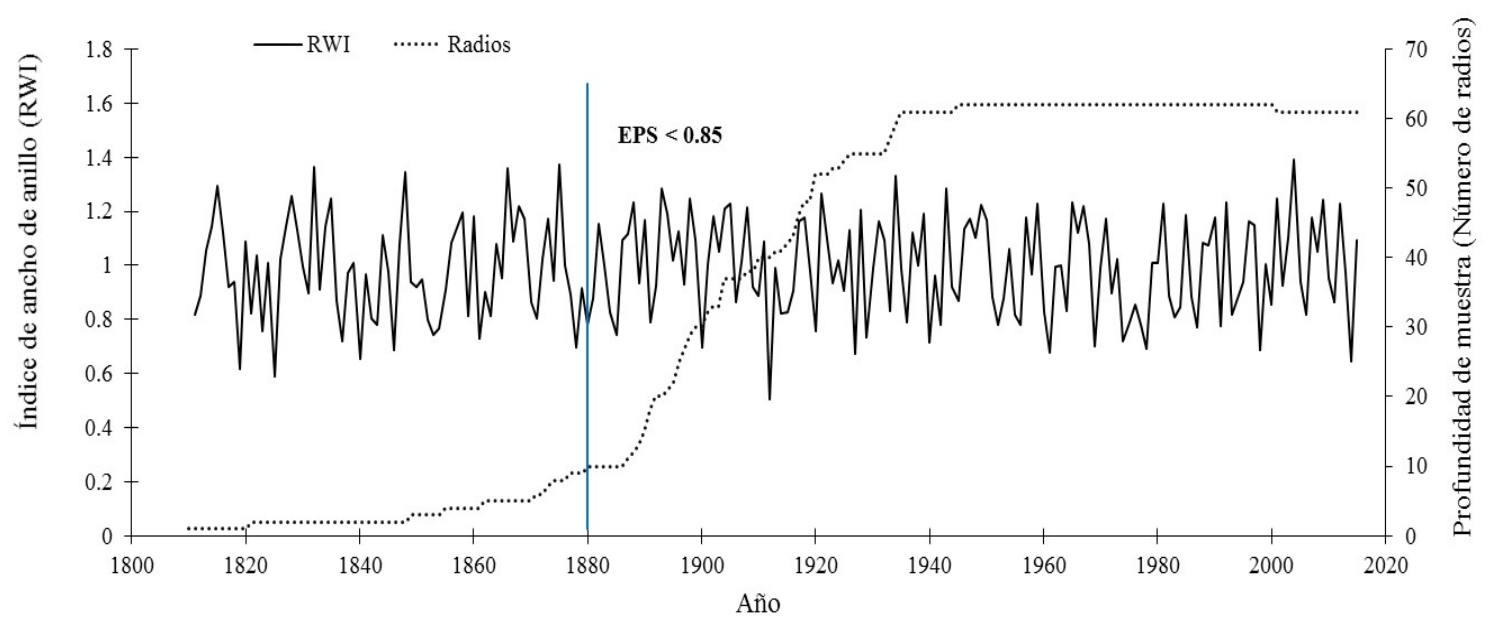

FIGURA 3. Cronología residual de anillo total (RWI) de Abies guatemalensis (línea continua) y tamaño de muestra (línea punteada). La línea en color azul indica la fecha a partir de donde la cronología rebasa el valor de EPS de 0.85. 

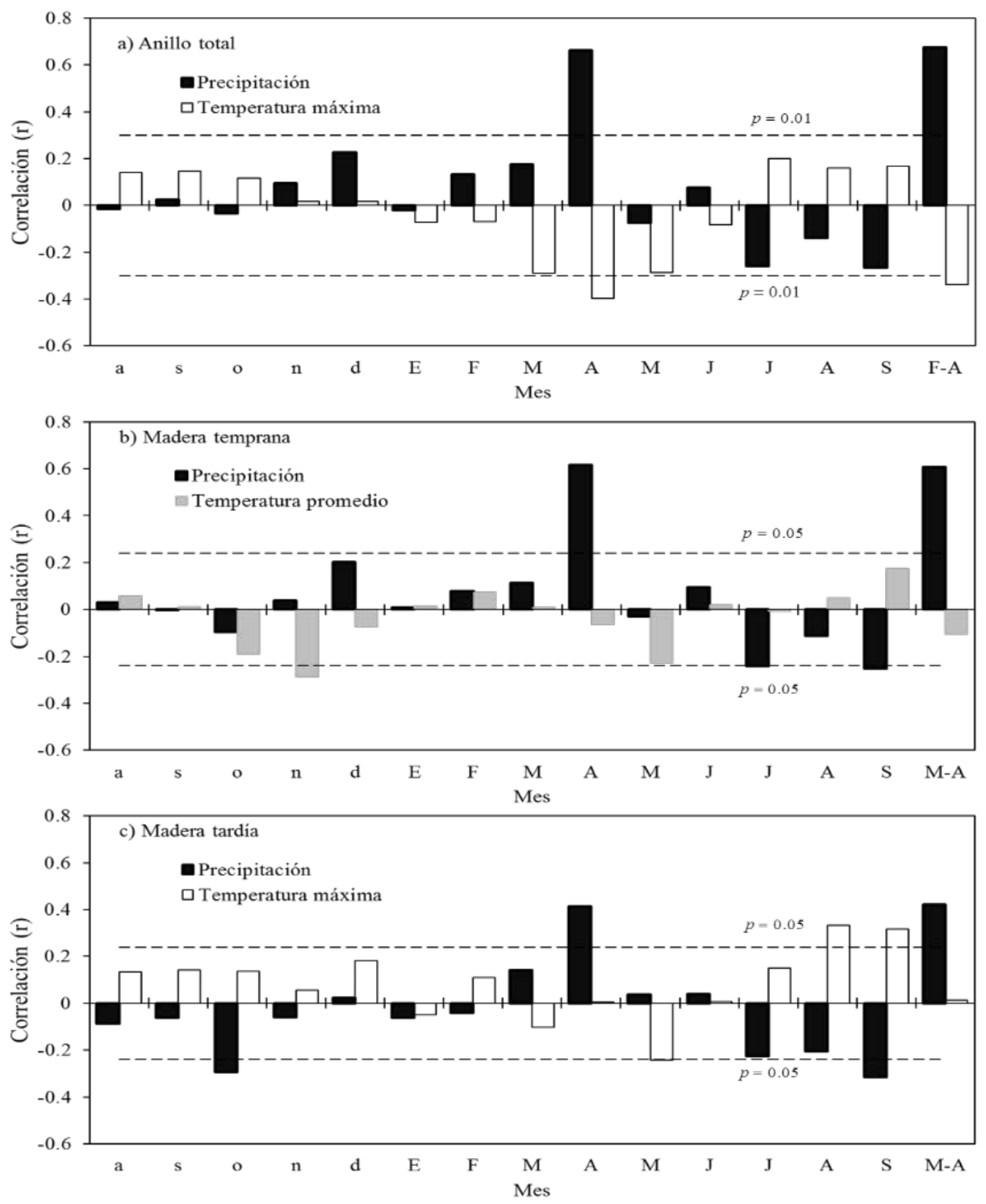

FIGURA 4. Coeficientes de correlación entre la cronología de anillo total versión residual (a), madera temprana versión residual (b) y madera tardía versión estándar (c) con la precipitación promedio, la temperatura promedio y la temperatura máxima, para el período 1952-2010.

Las líneas horizontales discontinuas indican los niveles de confianza a 95\% y 99\%. Las letras a-d en el eje horizontal representa los meses de agosto a diciembre del año previo y E-S los meses de enero a septiembre del año actual de crecimiento; F-A: febrero-abril, M-A: marzo-abril.

$(\mathrm{r}=0.33, \mathrm{p}<0.05)($ Tabla 1). El crecimiento del anillo total de los árboles está más asociado con la precipitación de febrero-abril $(\mathrm{r}=0.67, \mathrm{p}<0.01)$ y la temperatura máxima de marzo-mayo ( $\mathrm{r}=-0.42, \mathrm{p}<0.01)$, ambos del año actual (Fig. 4, Tabla 1). Por otro lado, la temperatura mínima no mostró ninguna asociación estadística significativa ( $\mathrm{p}>$ 0.05) con las cronologías de RWI, EWI y LWI.

\section{Reconstrucción de la precipitación}

Con base en los resultados obtenidos del análisis de correlación, la precipitación estacional de febrero-abril
( $\left.\mathrm{P}_{\mathrm{FMA}}\right)$ fue el factor climático más limitante en el crecimiento de $A$. guatemalensis. Por lo tanto, la cronología RWI residual fue elegida para la reconstrucción climática local de la $P_{\mathrm{FMA}}$ en Ixtlán de Juárez, Oaxaca. La ecuación desarrollada para reconstruir la precipitación acumulada de febrero-abril para Ixtlán de Juárez fue la siguiente:

$$
P_{F M A}=-36.2082+(94.5988 \times R W I)
$$


Donde:

$P_{F M A}$ : precipitación acumulada $(\mathrm{mm})$ de febrero-abril

$R W I$ : índice de ancho de anillo de la cronología residual

El modelo de regresión lineal desarrollado explica 44\%

de la variación de la PFMA para el período de 1952-2010
(Fig. 5). Los valores altos de $R^{2}(0.44)$ y F-test (46.29) indican la confiabilidad del modelo final. El valor de DW fue de 1.86 lo que reveló que no hay autocorrelación significativa en los residuales. La correlación entre la reconstrucción y la precipitación observada fue de 0.66 (Tabla 2).

TABLA 1. Respuesta climática entre los índices de madera temprana, tardía y de anillo total y las variables climáticas del período $1952-$ 2010.

\begin{tabular}{|c|c|c|c|c|}
\hline Cronología & Versión & Variable climática & Período mensual/estacional & Asociación \\
\hline \multirow[t]{7}{*}{ RWI } & Residual & Precipitación & abril & $r=0.66, p<0.01$ \\
\hline & & Precipitación & febrero-abril & $r=0.67, p<0.01$ \\
\hline & & Temperatura promedio & marzo & $r=-0.29, p<0.01$ \\
\hline & & Temperatura promedio & abril & $r=-0.39, p<0.01$ \\
\hline & & Temperatura promedio & mayo & $r=-0.28, p<0.01$ \\
\hline & & Temperatura promedio & febrero-abril & $r=-0.32, p<0.01$ \\
\hline & & Temperatura máxima & marzo-mayo & $r=-0.42, p<0.01$ \\
\hline \multirow[t]{3}{*}{ EWI } & Residual & Precipitación & abril & $r=0.61, p<0.05$ \\
\hline & & Precipitación & marzo-abril & $r=0.60, p<0.05$ \\
\hline & & Temperatura promedio & noviembre & $r=-0.28, p<0.05$ \\
\hline \multirow[t]{3}{*}{ LWI } & Residual & Precipitación & abril & $r=0.41, p<0.05$ \\
\hline & Estándar & Precipitación & marzo-abril & $r=0.60, p<0.05$ \\
\hline & Estándar & Temperatura máxima & agosto & $r=0.33, p<0.05$ \\
\hline
\end{tabular}

RWl: cronología estandarizada de anillo total; EWl: madera temprana; LWI: madera tardía.

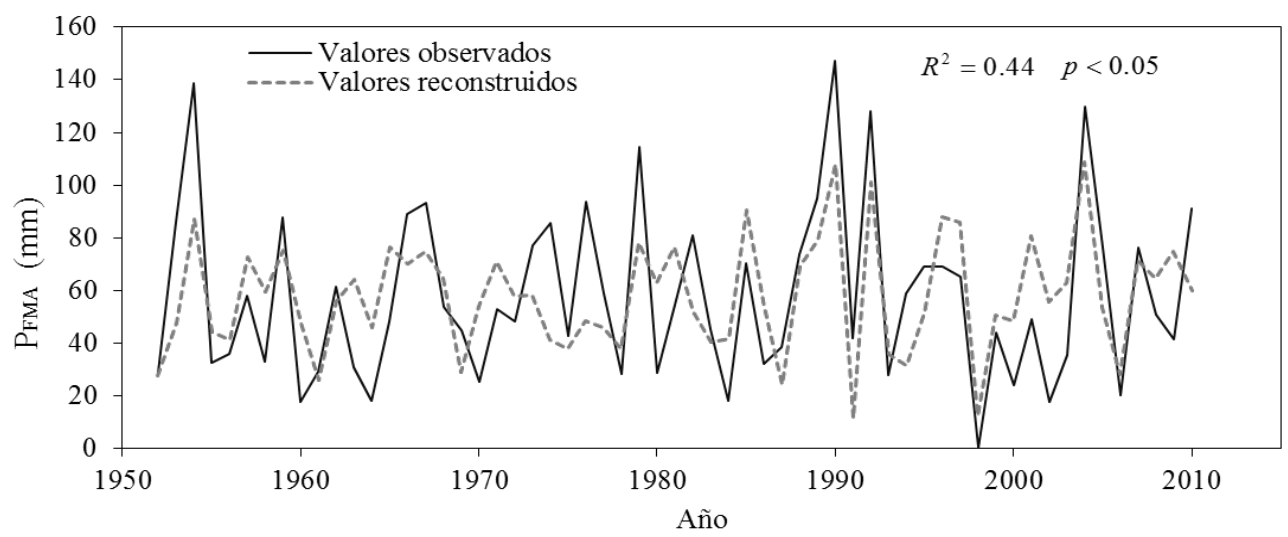

FIGURA 5. Comparación entre los valores observados y valores reconstruidos de la precipitación acumulada de febrero-abril (P el período 1952-2010, en Ixtlán de Juárez Oaxaca 
TABLA 2. Estadísticos para la calibración y verificación del modelo utilizado para la reconstrucción de la P PMA en el período de $1952-$ 2010.

\begin{tabular}{lllllllllll}
\hline Calibración & $r$ & $R^{2}$ & $R_{a d j}^{2}$ & $F$ & Verificación & $r$ & CE & $R E$ & $R M S E$ & DW \\
\hline $1952-1981$ & 0.54 & 0.29 & 0.27 & 11.55 & $1982-2010$ & 0.75 & 0.56 & 0.57 & 1.09 & 1.65 \\
$1981-2010$ & 0.74 & 0.55 & 0.53 & 34.86 & $1952-1980$ & 0.55 & 0.31 & 0.30 & 2.57 & 1.71 \\
$1952-2010$ & 0.66 & 0.44 & 0.43 & 46.29 & & & & & & 1.86 \\
\hline
\end{tabular}

$r$ : Coeficiente de correlación de Pearson;: $R^{2}$ coeficiente de determinación; $R^{2}$ adj: coeficiente de determinación ajustado; F: estadístico para la significancia del modelo de regresión; CE: coeficiente de eficiencia; RE: reducción del error; RMSE: raíz de los cuadrados medios del error; DW: Durbin-Watson.

La estabilidad y confiabilidad del modelo final fue validado usando pruebas de calibración y verificación entre los datos observados y reconstruidos. Los registros instrumentales de la precipitación (1952-2010) fueron subdivididos en dos partes: 30 años para calibración (1952-1981/1981-210) y 29 años para verificación (1982-2010/1952-1980) (Tabla 2). Los estadísticos r, CE, RE y DW en los subperíodos de verificación demostraron que el modelo usado para la reconstrucción es confiable y estable, por lo tanto, se consideró al período total de registros de precipitación para desarrollar el modelo final de reconstrucción. La reconstrucción permitió identificar la variación histórica local de la P PMA para el período de 1880-2015.

\section{Variabilidad de la precipitación reconstruida}

El valor promedio de la $P_{\text {FMA }}$ reconstruida fue de $57.59 \mathrm{~mm}$ con una desviación estándar $(\sigma)$ de $22 \mathrm{~mm}$. La reconstrucción de la $\mathrm{P}_{\mathrm{FMA}}$ muestra distintos períodos secos y húmedos durante los últimos 136 años (1880-2015) en la zona de Ixtlán de Juárez, Oaxaca. La reconstrucción presentó 18 años secos y 19 años húmedos, que representan $13.97 \%$ y $13.23 \%$ del total de años de la serie, respectivamente.

Los años más extremos de sequía fueron 1880, 1885, 1912, 1933, 1952, 1961, 1975, 1987, 1991, 1998, 2006 у 2014, y los años extremos de humedad fueron 1934,1954, 1985, 1990,1992 y 2004. Así como, los principales períodos de sequía ocurrieron en 1884-1885, 1900-1901, 1940-1942, 1951-1953, 1960-1961, 1974-1978, 1983-1984 y 1993-1994. Por el contrario, los períodos húmedos se registraron de 1986-1888, 1890-1891, 1893-1894, 1902-1905, 1917-1918,
1931-1932, 1937-1939, 1946-1950, 1965-1967, 1988-1990, 1996-1997 y 2007-2009 (Fig. 6).

En siglo XX las sequías más intensas ocurrieron en 1934-1935, 1940-1941, 1945-1950, 1954, 1957-1958, 1977 , 1983-1984 y 1987; las cuales coinciden con los años de sequías severas identificadas a través del índice de severidad de sequía de palmer (PDSI, por sus siglas en inglés) para el estado (Liverman, 1999) y los reconstruidos con el Atlas de sequías de México (MXDA) (Stahle et al., 2016) (Fig. 7). Los resultados del análisis espectral permitieron identificar la variabilidad de la P $_{\mathrm{MFA}}$ reconstruida (1880-2015). Los períodos significativos $(\mathrm{p}<0.01)$ fueron de 2.2 años y 3.8 años (Fig. 8).

\section{Influencia de fenómenos circulatorios}

La correlación entre los diferentes índices de ancho de anillo (EWI, LWI, RWI) de A. guatemalensis y los índices climáticos fueron bajos (Tabla 3). Los índices SST 3.4 y TRI expresaron una asociación negativa significativa con la cronología de anillo total versión residual para el período estacional febrero-marzo $(r=-0.365, \mathrm{p}<0.05)$ y para el mes de mayo $(\mathrm{r}=-0.191, \mathrm{p}<0.05)$, respectivamente. De igual manera, el índice MEI manifestó una relación negativa con la cronología de madera temprana versión estándar (EWI_STD) para el mes de febrero $(\mathrm{r}=-0.276, \mathrm{p}<0.05)$. Una correlación positiva se manifestó con AMO y la cronología de madera tardía versión residual (LWI_RES) para período estacional enero febrero $(r=0.259, \mathrm{p}<0.05)$. Por lo que respecta al PDO no se presentó asociación significativa con las series de anillo total para el mes de febrero $(r=-0.164, \mathrm{p}>0.05)$. 


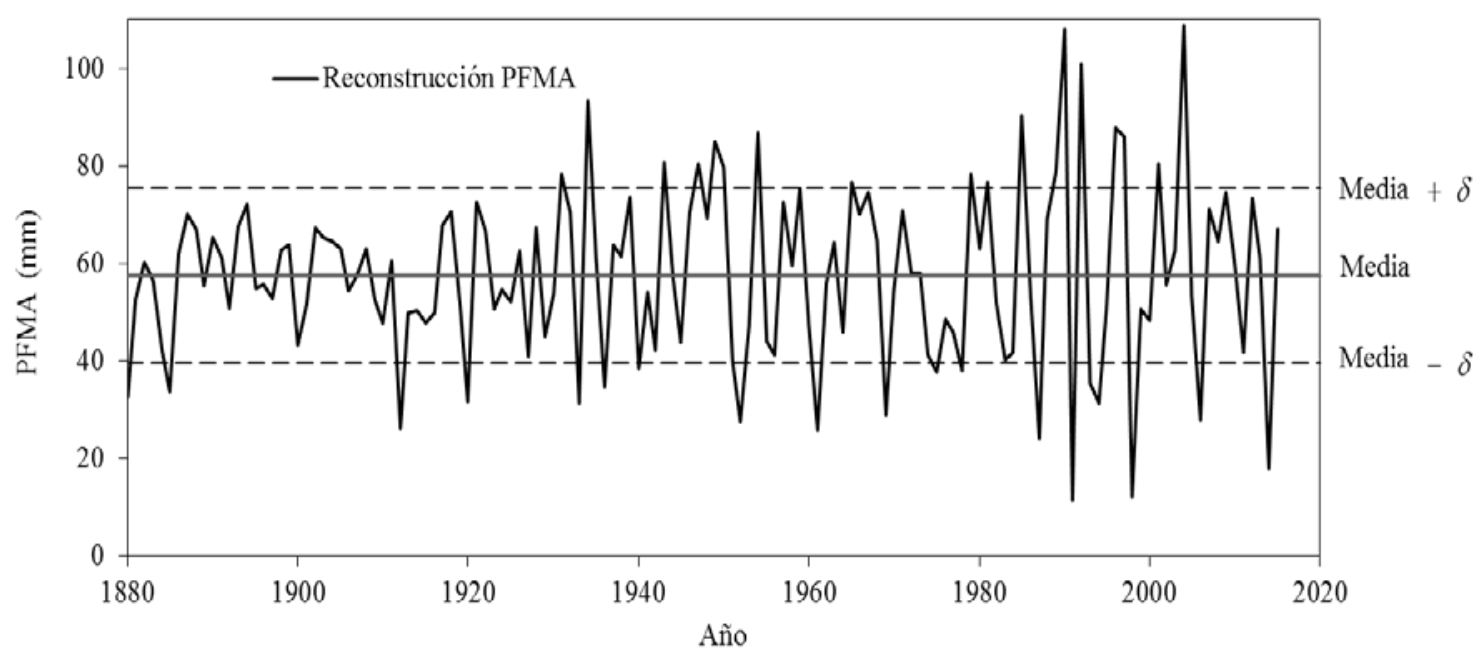

Figura 6. Reconstrucción de la $\mathrm{P}_{\mathrm{MFA}}$ en los últimos 136 años (1880-2015), en Ixtlán de Juárez Oaxaca.

Las líneas horizontales punteadas representan los umbrales para establecer los años secos y húmedos extremos, de acuerdo con la metodología propuesta por Sun et al. (2018).
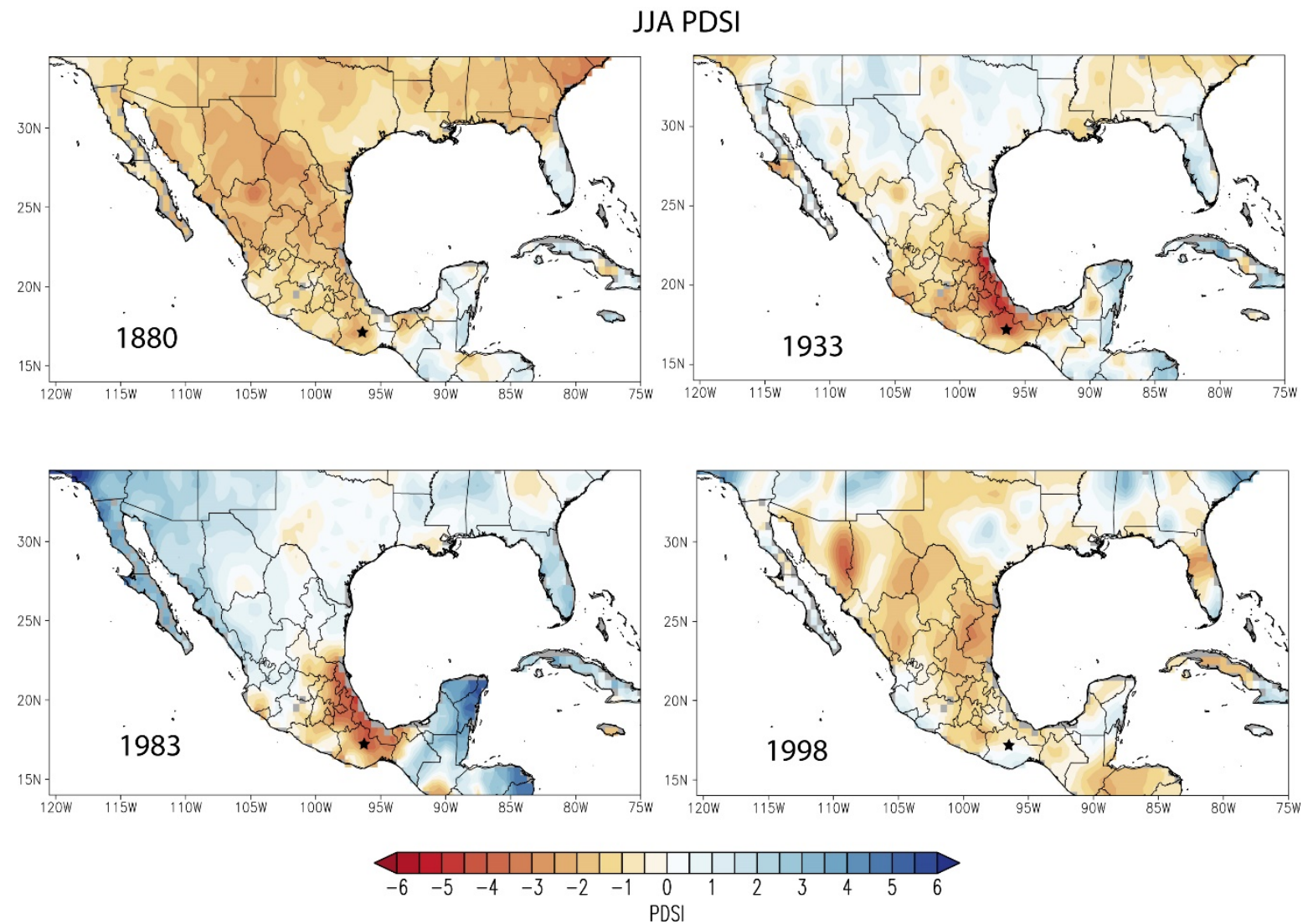

Figura 7. Mapas de sequías generados con el Atlas de sequía para México (MXDA) (Stahle et al., 2016). Condiciones de sequías en el sur de México y su coincidencia con los años más extremos de las sequías reconstruidas con Abies guatemalensis en Ixtlán de Juárez Oaxaca. Los valores -6 (color rojo) y 6 (color azul) en la escala PDSI indican condiciones de sequía extrema y humedad extrema. La estrella negra en los mapas muestra el área de estudio. 


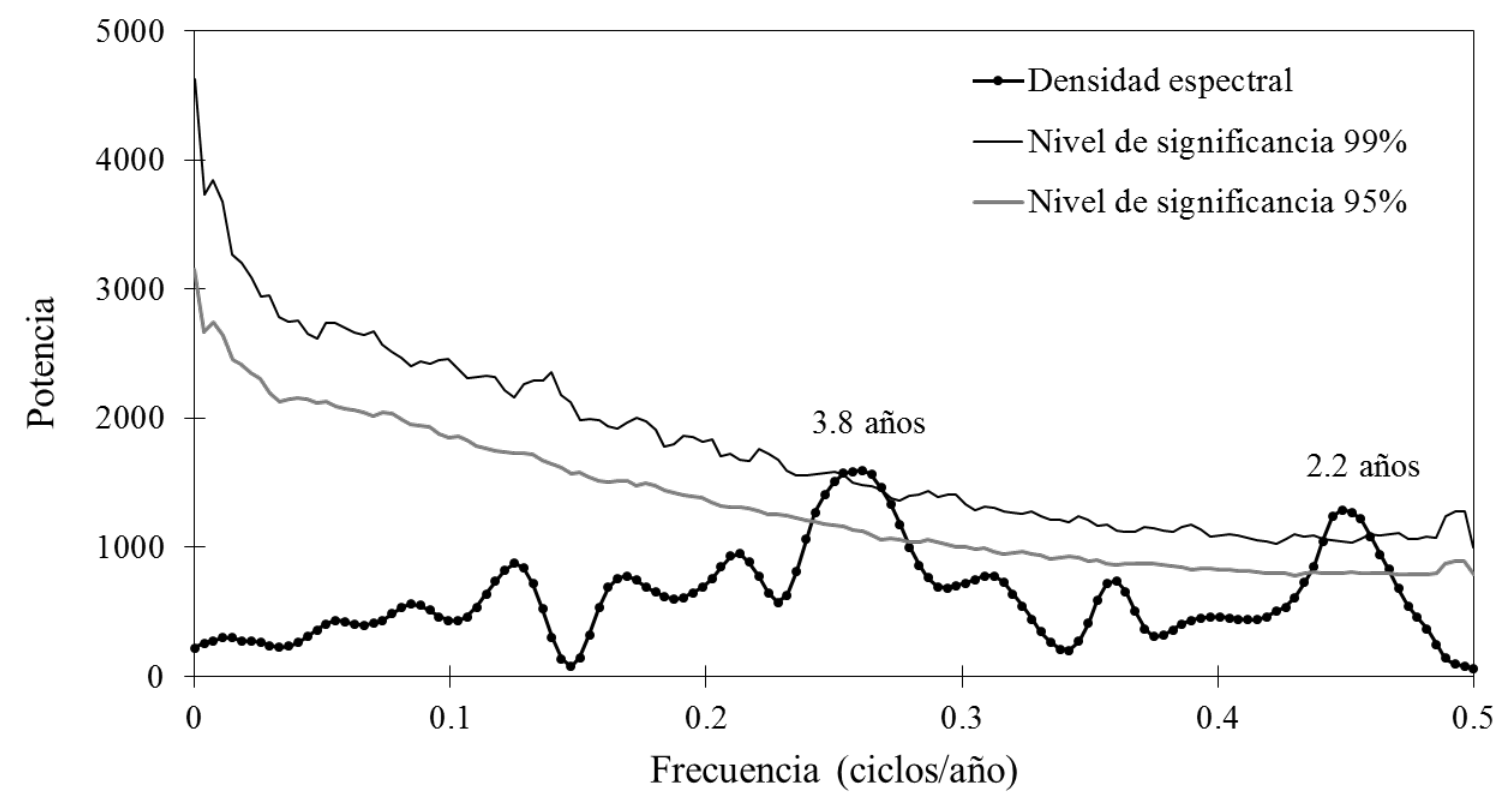

FIGURA 8. Densidad espectral para la reconstrucción de la PMFA (1880-2015) en la región de Ixtlán de Juárez, Oaxaca.

TABla 3. Asociación entre las cronologías de Abies guatemalensis en Ixtlán de Juárez Oaxaca y los diferentes índices de fenómenos de circulación global.

\begin{tabular}{llllll}
\hline $\begin{array}{l}\text { Fenómeno } \\
\text { circulatorio }\end{array}$ & Índice & Cronología & Período (años) & Meses & Correlación ( $p<0.05)$ \\
\hline \multirow{4}{*}{ ENSO } & SOI & RWI_RES & $1880-2015$ & febrero-mayo & -0.182 \\
& MEI & EWI_STD & $1880-2005$ & enero-febrero & -0.276 \\
& MEI & RWI_RES & $1880-2005$ & enero-febrero & -0.224 \\
& Niño 3.4 SST & RWI_RES & $1880-2015$ & febrero-marzo & -0.365 \\
PDO & Niño 3.4 SST & EWI_STD & $1880-2015$ & febrero & -0.248 \\
AMO & TRI & RWI_RES & $1894-1995$ & mayo & -0.191 \\
\hline
\end{tabular}

NS: no significativo.

\section{DISCUSIÓN}

Con base en los parámetros dendrocronológicos descritos, que manifiestan una señal climática común entre las series de A. guatemalensis, es posible catalogar a esta especie con potencial dendrocronológico para reconstrucciones climáticas. La correlación entre series y la sensibilidad media en este estudio fueron similares a lo obtenido por Anchukaitis et al. (2013) $(0.523,0.244)$ en A. guatemalensis en la Sierra de los Cuchumatanes, Guatemala y a los publicados por Huante, Rincón y Swetnam (1991) (0.55, 0.15); Cerano et al. (2014) (0.47, 0.37) y Carlón, Mendoza, Pérez-Salicrup, Villanueva-Díaz y Lara (2016) (0.54, 0.22) en $A$. religiosa en Michoacán, México. Sin embargo, estos parámetros son inferiores a los de Pseudotsuga men₹iesii (0.71, 0.37) (Cerano-Paredes et al., 2012) y Pinus cembroides (0.72, 0.30) (Constante-García, Villanueva-Díaz, Cerano-Paredes, Cornejo-Oviedo y Valencia-Manzo, 2009), especies reconocidas por su alto potencial dendrocronológico para reconstrucciones paleoclimáticas.

El crecimiento de $A$. guatemalensis tuvo una respuesta positiva a la precipitación acumulada entre febrero-abril; mientras que la respuesta fue negativa a la temperatura máxima alcanzada durante los meses de marzo-mayo. En consecuencia, si las condiciones de precipitación al finalizar 
el invierno y principios de la primavera no son las adecuadas, un aumento en la temperatura durante la estación de primavera incrementaría la evapotranspiración y disminuiría la humedad del suelo, limitando así en el crecimiento radial (Dawadi, Liang, Tian, Devkota y Yao, 2013; Thapa, Shah, Gaire y Bhuju, 2015). En cambio, un año con alta precipitación estacional febrero-abril favorecerá el crecimiento por su influencia directa en la disponibilidad de humedad del suelo, compensando así la pérdida de agua por el proceso de evapotranspiración ( $\mathrm{Li}$, Gou, Cook y Chen, 2006).

La respuesta climática obtenida de $A$. guatemalensis en esta investigación es consistente con la hallada por Anchukaitis et al. (2013) para la misma especie. Así mismo, diferentes estudios dendroclimáticos realizados para $A$. religiosa en Michoacán, México, han indicado una relación positiva con la precipitación invierno-primavera y una respuesta negativa con la temperatura invernal del año previo y la temperatura de primavera del año actual de crecimiento (Huante et al., 1991; Cerano et al., 2014; Carlón et al., 2016). Igualmente, otras especies de coníferas en el norte y noreste de México como P. menziesii (Villanueva et al., 2015), P. durangensis, P. cooperi y P. Lumboltziii (ChávezGándara et al., 2017) han mostrado una respuesta climática positiva a la precipitación invierno-primavera. La similitud en la respuesta climática mostrada por las coníferas en las diferentes regiones de México puede ser indicativo de que fenómenos climáticos de amplio impacto pueden provocar las condiciones climáticas dominantes que caractericen la variabilidad observada en dicho período estacional; uno de estos fenómenos de amplio alcance es ENSO (Stahle et al., 2011).

Los valores positivos de $\mathrm{CE}$ y $\mathrm{RE}$ en los dos subperíodos de verificación expresaron la potencia predictiva y la estabilidad del modelo de regresión (Cook et al., 1999). El valor del estadístico DW para el período completo fue cercano a 2 lo que revela que no hay autocorrelación significativa en los residuales anuales del modelo de regresión (Durbin y Watson, 1950). El modelo explicó 44\% de la variabilidad de la precipitación estacional febrero-abril, valor que fue superior al obtenido por Anchukaitis et al. (2015) en la reconstrucción de la precipitación estacional enero-marzo con anillos anuales de A. guatemalensis donde el modelo capturó $30 \%$ de la variabilidad.

Aunque la precipitación del período estacional reconstruido (febrero-abril), solo representa $6 \%$ del total anual, estos resultados coinciden con los períodos de sequía documentados en el estado de Oaxaca, como la ocurrida en 1885, registrada como una de las sequías más severas en el siglo XIX provocando pérdidas en las cosechas (Mendoza, Velasco y Jáuregui, 2006). Las sequías ocurridas en las décadas de 1940, 1950, 1960, 1970, que afectaron casi todo el país (Florescano, Sancho y Gavilán, 1980), también fueron registradas en otros estudios climáticos realizados en el norte y centro del país (Therrell, Stahle, Diaz, Oviedo y Cleaveland, 2006; Cerano et al., 2014; Villanueva et al., 2015). Otra sequía importante registrada en los últimos años de escala nacional fue la acontecida en 1998, misma que afecto la región oeste del país (Cerano et al., 2014) y la del 2011, calificada como la peor sequía en las últimas siete décadas para los estados del norte y centro del país, incidiendo en $60 \%$ del territorio mexicano (Domínguez, 2016). Al contrario, los años en que se presentaron lluvias atípicas fueron 1934, 1949, 1954, 1985, 1990 y 2004, mismas que fueron registradas en la reconstrucción.

Los períodos significativos de la reconstrucción $(2.2$ años y 3.8 años), se sitúan dentro de los picos espectrales (2 años - 8 años) que caracterizan la variabilidad de la oscilación del fenómeno de ENSO (Santoso, Mcphaden y Cai, 2017), sugiriendo una posible vinculación con la variabilidad interanual de la precipitación local. De la misma manera, los cuasi-ciclos de 2 años - 3 años podrían estar relacionados con la oscilación cuasi-bienal (Labitzke y Van Loon, 1999). La correlación entre el índice Niño 3.4 SST y la cronología residual del anillo total indicó una influencia de la precipitación estacional. En los años considerados como eventos de El Niño moderados (1963, 1968, 1976, 1977, 1987, 1994, 2002 y 2006) a intensos (1973, 1983, 1991 y 1998) (Santoso et al., 2017), la precipitación estacional 
febrero-abril disminuyó en $21.37 \%$ y $47.29 \%$ con respecto a la media $(57.59 \mathrm{~mm})$, en tanto que el crecimiento se redujo con un valor de índice de ancho de anillo anual menor al promedio $(<1.0)$.

Los ciclos de alta frecuencia asociados con ENSO, como uno de los factores que influyen en la variación local de sequías y humedad, han sido registrados en otras reconstrucciones de precipitación en el norte y centro de México (Cerano et al., 2014; Villanueva et al., 2015). Las anomalías secas y cálidas durante los eventos del Niño afectan el crecimiento de los árboles, de tal forma que favorecer una mayor presencia de lluvia en la estación invierno-primavera en el norte y condiciones de sequía durante el verano en centro y sur de México (Méndez y Magaña; 2010). El fenómeno climático de escala global que ha tenido importantes efectos en la precipitación de México es ENSO (Magaña, Vázquez, Pérez y Pérez, 2003); en la región sur del país, este fenómeno en su fase cálida (El Niño) tiene efectos negativos en la precipitación de verano y en su fase fría (La Niña) incrementa la precipitación de invierno. Estudios realizados en el sur de México sobre reconstrucciones de sequías han demostrado que la variabilidad climática en esta región está influenciada por patrones climáticos de gran escala en particular con ENSO. Mendoza et al. (2006) al correlacionar una serie de tiempo de sequías (1502-1899) con el SOI se observó que 38\% de las sequías en Oaxaca estaban relacionadas con eventos de El Niño. De igual forma, Liverman (1999) encontró que las sequías de 1957-1958, 1977, 1982-1983 y 1987 en Oaxaca están asociadas con los valores bajos del SOI, especialmente con los eventos del El Niño, donde las condiciones más secas que el promedio en el sur de Mexico ocurren durante los años en el que el SOI cambia de signo de positivo a negativo y cuando las anomalías en la temperatura de la superficie del mar del Pacifico ecuatorial y oriental se vuelven fuertemente positivas (Kiladis y Diaz, 1993).

Los ciclos multidecadales de baja frecuencia asociados a los fenómenos circulatorios globales como el PDO y AMO no fueron significativos en la reconstrucción, probablemente debido a la corta longitud de la serie reconstruida (136 años). La influencia del PDO ha sido registrada en el noroeste de México modificando la precipitación de primavera y verano; mientras que cada cambio en el comportamiento de AMO altera los patrones de la precipitación invernal en el noreste del país (AlvarezOlguin y Escalante-Sandoval, 2017).

\section{CONCLUSIONES}

Los parámetros dendrocronológicos obtenidos de las series de anillo total, de madera temprana y de madera tardía de Abies guatemalensis indicaron un fechado confiable y una señal de crecimiento común entre árboles. La precipitación de invierno-primavera y la temperatura máxima de primavera fueron las variables climáticas que afectaron el incremento radial anual de Abies guatemalensis. La serie dendrocronológica desarrollada permitió reconstruir la variabilidad interanual de la precipitación de febrero-abril, resaltando los períodos secos y húmedos en la zona de Ixtlán, Oaxaca durante los últimos 136 años (1880-2015). Las sequías en esta región de México están vinculadas con los sistemas de circulación atmosférica como ENSO.

La sensibilidad interanual y multianual de los índices de ancho de anillo y su asociación con variables climáticas como precipitación y temperatura indican que $A$. guatemalensis tiene potencial para reconstruir la variabilidad hidroclimática del pasado más allá de los datos instrumentales disponibles. Esta información es importante para los administradores y planificadores de los recursos naturales, estudios hidrológicos y ecológicos. Las reconstrucciones realizadas con especies del genero Abies permitió analizar con mayor detalle la intensidad y extensión de fenómenos circulatorios que afectan esta región de México.

\section{RECONOCIMIENTOS}

Se agradece a las autoridades del Comisariado de Bienes Comunales de Ixtlán de Juárez por las facilidades otorgadas y al técnico forestal responsable, M. C. Elías Santiago García, el apoyo para la toma de datos.

\section{REFERENCIAS}

Acosta-Hernández, A. C., Pompa-García, M., \& Camarero, J. J. (2017). An updated review of dendrochronological investigations in Mexico, a megadiverse country with a high potential for tree-ring sciences. Forests, 8(5), 160. doi: $10.3390 /$ f8050160

Alvarez-Olguin, G., \& Escalante-Sandoval, C. (2017). Modes of variability of annual and seasonal rainfall in 
Mexico. JAWRA Journal of the American Water Resources Association, 53(1), 144-157. doi: 10.1111/1752-1688.12488

Anchukaitis, K. J., Taylor, M. J., Leland, C., Pons, D., MartinFernandez, J., \& Castellanos, E. (2015). Tree-ring reconstructed dry season rainfall in Guatemala. Climate dynamics, 45(5-6), 1537-1546.

Anchukaitis, K. J., Taylor, M. J., Martin-Fernandez, J., Pons, D., Dell, M., Chopp, C., \& Castellanos, E. J. (2013). Annual chronology and climate response in Abies guatemalensis Rehder (Pinaceae) in Central America. The Holocene, 23(2), 270-277. doi: 10.1177/0959683612455548

Bernal-Salazar, S., Terrazas, T., y Alvarado, D. (2004). Impact of air pollution on ring width and tracheid dimensions in Abies religiosa in the Mexico City basin. Iawa Journal, 25(2), 205215. doi: 10.1163/22941932-90000361

Bunn, A. G. (2010). Statistical and visual crossdating in R using the dplR library. Dendrochronologia, 28(4), 251-258. doi: 10.1016/j.dendro.2009.12.001

Carlón A. T., Mendoza, M. E., Pérez-Salicrup, D. R., VillanuevaDíaz, J., \& Lara, A. (2016). Climatic responses of Pinus pseudostrobus and Abies religiosa in the monarch butterfly biosphere reserve, central Mexico. Dendrochronologia, 38, 103116. doi: 10.1016/j.dendro.2016.04.002

Cerano J. P., Díaz, J. V., Martínez, R. C., Selem, L. V., Caciano, R. T., \& De la Cruz, V. G. (2014). Reconstrucción de precipitación invierno-primavera para el Parque Nacional Pico de Tancítaro, Michoacán. Investigaciones Geográficas, Boletín del Instituto de Geografía, 2014(83), 41-54. doi: 10.14350/rig. 35190

Cerano-Paredes, J., Villanueva-Díaz, J., Valdez-Cepeda, R. D., Constante-García, V., González-Barrios, J. L., \& EstradaÁvalos, J. (2012). Precipitación reconstruida para la parte alta de la cuenca del río Nazas, Durango. Revista Mexicana de Ciencias Forestales, 2(10), 7-23.

Chávez-Gándara, M. P., Cerano-Paredes, J., Nájera-Luna, J. A., Pereda-Breceda, V., Esquivel-Arriaga, G., CervantesMartínez, R., Cambrón-Sandoval, V. H., Cruz-Cobos, F. \& Corral-Rivas, S. (2017). Reconstrucción de la precipitación invierno-primavera con base en anillos de crecimiento de árboles para la región de San Dimas, Durango, México. Bosque (Valdivia), 38(2), 387-399. doi: $10.4067 /$ S0717-92002017000200016

Constante-García, V., Villanueva-Díaz, J., Cerano-Paredes, J., Cornejo-Oviedo, E. H., \& Valencia-Manzo, S. (2009). Dendrocronología de Pinus cembroides Zucc. y reconstrucción de precipitación estacional para el Sureste de Coahuila. Ciencia Forestal en México, 34(106), 17-39.
Cook, E. R. (1987). The decomposition of tree-ring series for environmental studies. Tree-Ring Bulletin, 47, 37-59.

Cook, E. R., Meko, D. M., Stahle, D. W., \& Cleaveland, M. K. (1999). Drought reconstructions for the continental United States. Journal of Climate, 12(4), 1145-1162. doi: 10.1175/1520-0442(1999)012<1145:DRFTCU>2.0.CO;2

Dawadi, B., Liang, E., Tian, L., Devkota, L. P., \& Yao, T. (2013). Pre-monsoon precipitation signal in tree rings of timberline Betula utilis in the central Himalayas. Quaternary International, 283, 72-77. doi: 10.1016/j.quaint.2012.05.039

Domínguez, J. (2016). Revisión histórica de las sequías en México: de la explicación divina a la incorporación de la ciencia. Tecnología y ciencias del agua, 7(5), 77-93.

Durbin, J., \& Watson, G. S. (1950). Testing for serial correlation in least squares regression: I. Biometrika, 37(3/4), 409-428.

Florescano M. E., Sancho J. C., \& Gavilán D. P.1980. Las sequías en México: historia, características y efectos. Comercio Exterior, 30, 747-757.

Frank, D., Esper, J., Zorita, E., \& Wilson, R. (2010). A noodle, hockey stick, and spaghetti plate: a perspective on highresolution paleoclimatology. Wiley Interdisciplinary Reviews: Climate Change, 1(4), 507-516. doi: 10.1002/wcc.53

Gaire, N. P., Bhuju, D. R., Koirala, M., Shah, S. K., Carrer, M., \& Timilsena, R. (2017). Tree-ring based spring precipitation reconstruction in western Nepal Himalaya since AD 1840. Dendrochronologia, 42, 21-30. doi: 10.1016/j.dendro.2016.12.004

García, E. (2004). Modificaciones al sistema de clasificación climática de Köppen. Ciudad de México, México: Instituto de Geografía, UNAM.

Hare, S. R. \& Mantua, N. J. (2000). Empirical evidence of North Pacific regime shifts in 1977 and 1989. Progress in Oceanography, 47(2-4), 103-145. doi: 10.1016/S00796611(00)00033-1

Holmes, R. L. (1983). Computer-assisted quality control in treering dating and measurement. Tree-Ring Bulletin, 43, 69-78.

Huante, P., Rincón, E., \& Swetnam, T. W. (1991). Dendrochronology of Abies religiosa in Michoacan, Mexico. Tree-Ring Bulletin, 51, 15-28.

Instituto Mexicano de Tecnología del Agua [IMTA] (2013). Estaciones climáticas extraído de ERIC III (Extractor Rápido De Información Climática). Jiutepec, Morelos, México: Instituto Mexicano de Tecnología del AguaSecretaría del Medio Ambiente y Recursos Naturales. 
Instituto Nacional de Estadística y Geografía [negi] (2010). Compendio de información geográfica municipal Ixtlán de Juárer, Oaxaca. Recuperado de http://www.inegi.org.mx/geo/contenidos/topografia/co mpendio.aspx

Islebe, G. A., Velázquez, A., \& Cleef, A. M. (1995). High elevation coniferous vegetation of Guatemala. Vegetatio, 116 (1), 7-23.

Kiladis, G. N. \& Diaz, H. F. 1993. ENSO and precipitation variability over Mexico during the last 90 years. En Redmond, K. T. \& Tharp, V L. (eds), Proceedings of the ninth annual pacific climate workshop (PACLIM) Workshop, 21-24 April, 1992, California Department of Water Resources, Interagency Ecological Studies Program, Technical Report 34.

Labitzke, K. G., \& Van Loon, H. (2012). The stratosphere: phenomena, history, and relevance. Springer: Berlin.180 p.

Li, J., Gou, X., Cook, E. R., \& Chen, F. (2006). Tree-ring based drought reconstruction for the central Tien Shan area in northwest China. Geophysical Research Letters, 33(7). doi: 10.1029/2006GL025803

Liverman, D. M. (1999). Vulnerability and adaptation to drought in Mexico. Natural Resources Journal, 39(1), 99-115.

Magaña, V. O., Vázquez, J. L., Pérez, J. L., \& Pérez, J. B. (2003). Impact of El Niño on precipitation in Mexico. Geofísica internacional, 42(3), 313-330.

Mann, M. E., \& Lees, J. M. (1996). Robust estimation of background noise and signal detection in climatic time series. Climatic change, 33(3), 409-445.

Mantua, N. J., Hare, S. R., Zhang, Y., Wallace, J. M., \& Francis, R. C. (1997). A Pacific interdecadal climate oscillation with impacts on salmon production. Bulletin of the American Meteorological Society, 78, 1069-1079. doi: 10.1175/15200477(1997)07811069:AP1COW>2.0.CO:2

Méndez, M., \& Magaña, V. (2010). Regional aspects of prolonged meteorological droughts over Mexico and Central America. Journal of Climate, 23(5), 1175-1188.

Mendoza, B., Velasco, V., \& Jáuregui, E. (2006). A study of historical droughts in southeastern Mexico. Journal of Climate, 19(12), 2916-2934. doi: 10.1175/JCLI3726.1

Pérez-Morga, N., Kretzschmar, T., Cavazos, T., Smith, S. V., \& Munoz-Arriola, F. (2013). Variability of extreme precipitation in coastal river basins of the southern Mexican Pacific region. Geofísica internacional, 52(3), 277-291. doi: 10.1016/S0016-7169(13)71477-6
R Development Core Team (2017). R: a Language and Environment for Statistical Computing. R Foundation for Statistical Computing, Vienna, Austria.

Ramírez, M. N., \& González E. M. (2015). Distribución, variación morfológica-genética, manejo y conservación de Abies guatemalensis. En M.R. Pineda L., L.R. Sánchez V. \& J.C. Noa-Carrazana (Eds.), Ecologia, biotecnologia y conservación del género Abies en México (pp. 186-205). Saarbrücken, Alemania: Editorial Académica Española.

Rayner, N. A., Parker, D. E., Horton, E. B., Folland, C. K., Alexander, L. V., Rowell, D. P., Kent, C. E., \& Kaplan, A. (2003). Global analyses of sea surface temperature, sea ice, and night marine air temperature since the late nineteenth century. Journal of Geophysical Research: Atmospheres, 108(D14). doi: 10.1029/2002JD002670

Ropelewski, C. F., \& Jones, P. D. (1987). An extension of the Tahiti-Darwin southern oscillation index. Monthly Weather Review, 115(9), 2161-2165.

Santoso, A., Mcphaden, M. J., \& Cai, W. (2017). The defining characteristics of ENSO extremes and the strong 2015/2016 El Niño. Reviens of Geophysics, 55(4), 1079-1129. doi: 10.1002/2017RG000560

Speer, J. H. (2010). Fundamentals of tree-ring research. University of Arizona Press. Tucson, AZ.

Stahle, D. W., Villanueva D. J., Burnette, D. J., Cerano P. J., Heim, R. R., Fye, F. K., Acuna S., Therrell M. D. Cleaveland, M.K. \& Stahle, D. K. (2011). Major Mesoamerican droughts of the past millennium. Geophysical Research Letters, 38(5). doi: 10.1029/2010GL046472

Stahle, D. W., Cook, E. R., Burnette, D. J., Villanueva, J., Cerano, J., Burns, J. N., Griffin, D., Cook, B. J., Acuña, R., Torbenson, M. C. A, Sjezner, P. \& Howard, J. M. (2016). The Mexican drought atlas: tree-ring reconstructions of the soil moisture balance during the late pre-Hispanic, colonial, and modern eras. Quaternary Science Review, 149, 34-60. doi: 10.1016/j.quascirev.2016.06.018

Sun, C., Liu, Y., Song, H., Mei, R., Payomrat, P., Wang, L., \& Liu, R. (2018). Tree-ring-based precipitation reconstruction in the source region of Weihe River, northwest China since AD 1810. International Journal of Climatology, 38(8), 3421-3431. Doi: $10.1002 /$ joc. 5514

Thapa, U. K., Shah, S. K., Gaire, N. P., \& Bhuju, D. R. (2015). Spring temperatures in the far-western Nepal Himalaya since AD 1640 reconstructed from Picea smithiana tree-ring widths. Climate dynamics, 45(7-8), 2069-2081. doi: $10.1007 / \mathrm{s} 00382-014-2457-1$ 
Therrell, M. D., Stahle, D. W., Diaz, J. V., Oviedo, E. H. C., \& Cleaveland, M. K. (2006). Tree-ring reconstructed maize yield in central Mexico: 1474-2001. Climatic Change, 74(4), 493. doi: 10.1007/s10584-006-6865-z

Van Oldenborgh, G. J., Te Raa, L. A., Dijkstra, H. A. \& Philip, S. Y. (2009). Frequency- or amplitude-dependent effects of the Atlantic meridional overturning on the tropical Pacific Ocean. Ocean Science, 5(3), 293-301. doi: 10.5194/os-5-2932009

Villanueva, D. J., Cerano, P. J., Fulé, P. Z., Cortés, M. C., Vázquez, S. L., Yocom, L. L., \& Ruiz-Corral, J. A. (2015). Cuatro siglos de variabilidad hidroclimática en el noroeste de Chihuahua, México, reconstruida con anillos de árboles. Investigaciones geográficas, (87), 141-153. doi: $10.14350 /$ rig.44485

Wigley, T. M., Briffa, K. R., \& Jones, P. D. (1984). On the average value of correlated time series, with applications in dendroclimatology and hydrometeorology. Journal of climate and Applied Meteorology, 23(2), 201-213.

Wolter, K., \& Timlin, M. S. (1998). Measuring the strength of ENSO events: How does 1997/98 rank?. Weather, 53(9), 315-324. doi: 10.1002/j.1477-8696.1998.tb06408.xWright, P. B. (1979). Persistence of rainfall anomalies in the central Pacific. Nature, 277(5695), 371. doi: 10.1038/277371a0
Zang, C., \& Biondi, F. (2015). Treeclim: an R package for the numerical calibration of proxy-climate relationships. Ecography, 38(4), 431-436. doi: 10.1111/ecog.01335

Zhang, Z. (2015). Tree-rings, a key ecological indicator of environment and climate change. Ecological indicators, 51, 107-116. doi: 10.1016/j.ecolind.2014.07.042

Manuscrito recibido el 15 de mayo de 2018

Aceptado el 8 de octubre de 2018

Publicado el 29 de julio de 2019

Este documento se debe citar como:

Aquino-Ramírez, M., Velázquez-Martínez, A., Villanueva-Díaz, J., Hervert-Zamora, H. L., Gómez-Guerrero, A., Reyes-Hernández, V. J., \& Ramírez-Valverde, G. (2019). Respuesta climática de Abies guatemalensis Rehder en Ixtlán de Juárez, Oaxaca, México. Madera y Bosques, 25(2), e2521773. doi: 10.21829/myb.2019.2521773

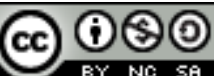

Madera y Bosques por Instituto de Ecología, A.C. se distribuye bajo una Licencia Creative Commons Atribución-NoComercialCompartirlgual 4.0 Internacional. 